\title{
Feds update immunization advice with Moderna vaccine approval
}

\author{
Cite as: CMAJ 2020 January 18;193:E108-9. doi: 10.1503/cmaj.1095914
}

Posted on cmajnews.com on December 23, 2020

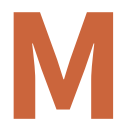
oderna and Pfizer COVID-19 vaccines are similar enough that you can swap them in a pinch, according to guidance from Canada's National Advisory Committee on Immunization (NACl).

The federal advisory group has updated its recommendations on COVID-19 vaccines to include advice on Moderna's shot, as well as more detailed information on immunizing people with allergies and other special medical circumstances.

Both the Moderna and Pfizer vaccines use similar mRNA technology, which gives the body genetic instructions to mount an immune response, and both are more than $90 \%$ effective after two doses, according to Dr. Caroline Quach of NACl. In a Phase III trial of the Moderna vaccine involving more than 30000 adults, there were 11 cases of COVID-19 among people who got the vaccine versus 185 among those who did not, and no cases of severe disease versus 30 in the placebo group.

Given a lack of data on the interchangeability of COVID-19 vaccines, NACI recommends that people receive the same vaccine product for both doses. But when that's not an option, patients who receive the Pfizer vaccine first could finish their series with the Moderna product, or vice versa. "Let's say you run out of Pfizer's vaccine because you've administered all your doses, or you don't know what your patient had at first... this eases up the complexity," says Quach.

\section{Are two doses essential?}

After one dose, both vaccines are $80 \%-$ $90 \%$ effective, but that protection may only last four to six weeks until a person gets their second dose. While Quach says that's "pretty good," NACl recommends that people complete a two-dose series of either vaccine within 28 days.

The guidance document unpacks ethical considerations for provinces weighing whether they should use their limited supplies of vaccines to give a first dose to as many people as possible right away, or keep half in reserve to ensure that people can get their second dose on time.

According to Quach, "some provinces and territories might want to manage their risk differently," and will need to consider the risks and benefits of wider, immediate protection versus a slower rollout of potentially longer lasting coverage. "We're happy to see that one dose is efficacious in the short term, but we don't know more than that. And we don't even know how long a two-dose series is going to be efficacious, so there's still quite a lot of uncertainty and we have to be transparent about it."

\section{New safety details}

Both vaccines are very safe but people who have severe allergies to components of the vaccines or their packaging should not be vaccinated. So far, the United Kingdom and United States have documented a handful of severe allergic reactions to the Pfizer vaccine. According to Quach, the key allergen in such cases appears to be polyethylene glycol - a stabilizing ingredient in both the Pfizer and Moderna vaccines that's also used in laxatives, cough syrup, cosmetics and some foods.

However, other people with allergies unrelated to the vaccine packaging or components can safely receive the shots.

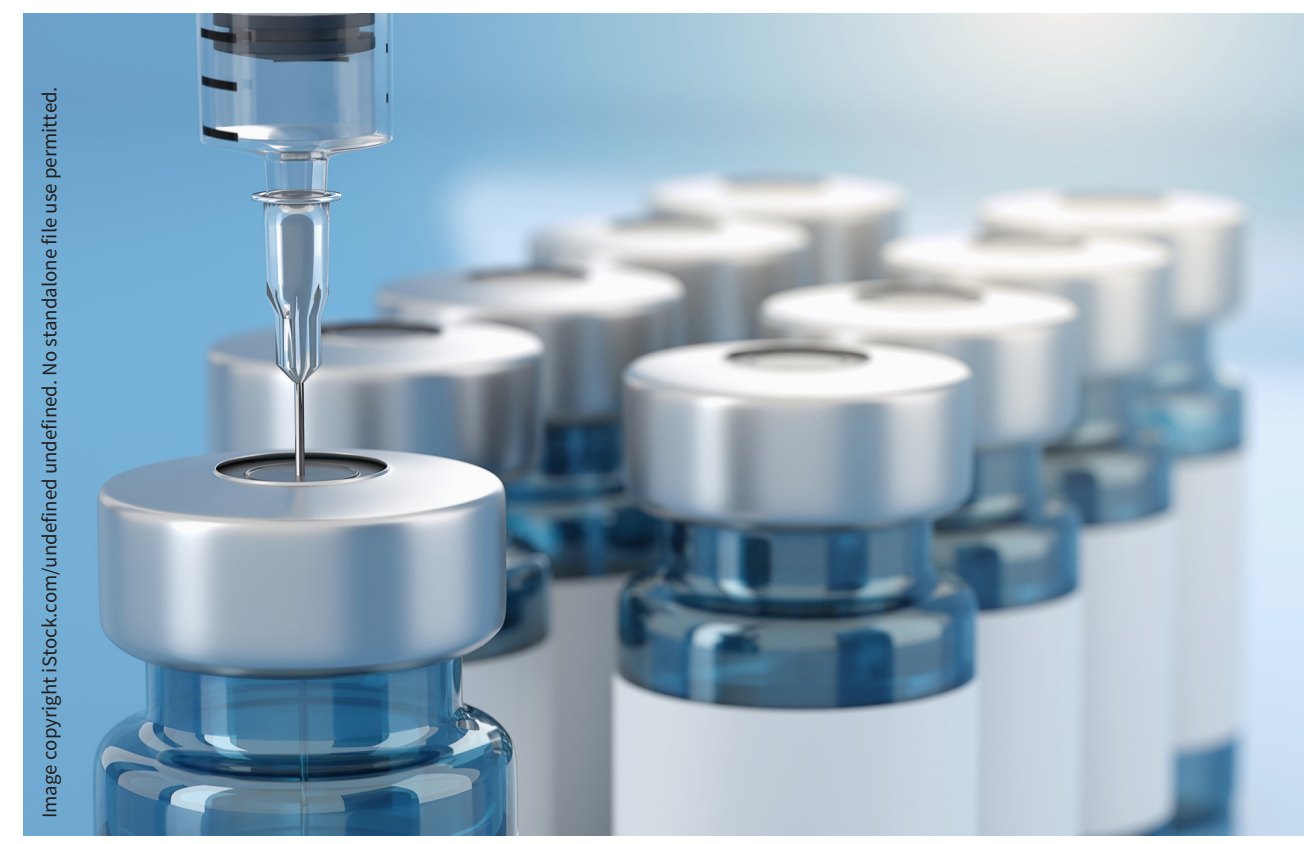

National recommendations on COVID-19 immunizations now include guidance on the safety, efficacy and use of both Moderna and Pfizer vaccines. 
For example, "being allergic to peanuts is not a risk factor," Quach says. Even if someone does experience an unexpected allergic reaction, the risk of serious harm is low because vaccinations are happening in medical settings where those reactions can be treated immediately, she explains.

Although long-term safety data for the Moderna and Pfizer COVID-19 vaccines are still pending, complications from immunizations typically develop "within four to six weeks," Quach notes. "We have 14 weeks of follow-up" supporting the safety of the vaccines.

Most people who get the Moderna or Pfizer vaccine will feel some discomfort for one or two days afterwards, which may include "feeling tired, having some pain at the injection site, having a little bit of fever," Quach explains. "You're going to feel it, but it's normal and benign to moderate. You don't have to stay in bed for it."

\section{Pregnancy and autoimmune issues} $\mathrm{NACl}$ also updated their guidance for people who are pregnant or breastfeeding, and people who are immunosupressed or have autoimmune diseases. "Some people have decided not to give the [Pfizer] vaccine to these populations because we said it shouldn't be administered routinely. But it's still available on a case by case basis," Quach says.

In the case of pregnant women, $\mathrm{NACl}$ has advised against routine vaccination because there is a "complete absence of data" until vaccine trial participants who were pregnant deliver their babies, Quach explains.

However, if a clinician thinks that a pregnant patient would benefit from vaccination against COVID-19 - for example, if she has a high risk of exposure or complications "you're absolutely able to recommend it," she says. "We don't think there's any particular problem, but we need data before saying it's a strong recommendation."

Likewise, people whose immune systems are suppressed should be considered for COVID-19 vaccines on a case by case basis, pending further evidence. According to Quach, NACl's main concern isn't safety, but that the vaccines won't work for people who are immunocompromised.

There's also a possibility that mRNA technologies may exacerbate inflammation and existing autoimmune disease. NACl's guidance document notes two cases of autoimmune diseases among a total of seven severe adverse events linked to the Moderna vaccine, both in patients with hypothyroidism. While the benefits of vaccination may outweigh the "theoretical risk" for some patients with existing autoimmune issues, "we need to monitor and make sure that's not a real risk," Quach says.

\section{Lauren Vogel, CMAJ}

Content licence: This is an Open Access article distributed in accordance with the terms of the Creative Commons Attribution (CC BY-NC-ND 4.0) licence, which permits use, distribution and reproduction in any medium, provided that the original publication is properly cited, the use is noncommercial (i.e., research or educational use), and no modifications or adaptations are made. See: https://creativecommons.org/ licenses/by-nc-nd/4.0/ 